\title{
Corelation of Perceived Social Support and Psychological Hardness with Perceived Stress and Aggression in Retired Veterans
}

\section{ART ICLE INF O}

\section{Article Type}

Descriptive Study

\section{Authors}

Ebrahimi L.*PhD,

Moradi $\mathrm{F}^{1} M A$

\section{How to cite this article}

Ebrahimi L, Moradi F. Corelation of Perceived Social Support and Psychological Hardness with Perceived Stress and Aggression in Retired Veterans. Iranian Journal of War \& Public Health. 2018;10 (3):157-164.
*Psychology Department, Humanities Faculty, University of Zanjan, Zanjan, Iran

${ }^{1}$ Clinical Psychology Department, Psychology Faculty, Zajan Branch, Islamic Azad University, Zanjan, Iran

\section{Correspondence}

Address: Room 38, Psychology Department, Humanities Faculty, University of Zanjan, Daneshgah Boulevard, Zanjan, Iran. Postal Code: 4537138791

Phone: +98 (24) 33054478

Fax: +98 (24) 33054181

l.ebrahimi@znu.ac.ir

\section{Article History}

Received: January 06, 2018

Accepted: March 14, 2018

ePublished: August 29, 2018

\section{A B S T R A C T}

Aims The purpose of this study was to investigate the role of perceived social support and psychological hardness in perceived stress and aggression of retired veterans.

Instruments \& Methods This study is a descriptive correlational which was done in Bijar city in 2017. The statistical population of the study consisted of all retired veterans of Bijar city at 92 samples were selected through an available sampling method based on Morgan's table and responded to perceived social support, hardness, perceived stress and aggression questionnaires. Data were analyzed by Pearson correlation, multiple regressions and SPSS 22 software.

Findings There was a significant negative corelation between perceived social support and perceived stress $(\mathrm{r}=-0.26 ; \mathrm{p}<0.05)$, perceived social support and aggression $(\mathrm{r}=-0.22 ; \mathrm{p}<0.05)$, Also, there was a negative and significant corelation between perceived stress and perceived stress $(\mathrm{r}=-0.30 ; \mathrm{p}<0.05)$, hardness and aggression $(\mathrm{r}=-0.27 ; \mathrm{p}<0.05)$. Finally, there was a significant positive correlation between perceived stress and aggression $(r=0.52 ; \mathrm{p}<0.05)$. Perceived social support and psychological hardness were able to significantly affect $33 \%$ of the variance of perceived stress in retired Veterans and significantly predict $49 \%$ of the variance of aggression in retired Veterans variable.

Conclusion There is a correlation between perceived social support and psychological Hardness with perceived stress and aggression of retired veterans, in a way that perceived social support and psychological hardness can be effective in reducing the stress and aggression of retired veterans and should be considered as effective moderating factors in stress and aggression.

Keywords Social Support; Hardness; Psychological Stresses; Aggression; Veterans

\section{I T A T I O N L I N K S}

[1] Compare elderly mental health nursing ... [2] Handbook of gerontology ... [3] Quality of life in elderly women ... [4] The retirement syndrome: The psychology ... [5] A study on cognitive and depressive ... [6] What cognitive changes can ... [7] Severly stressful events and dementia... [8] Comparative investigation of mental ... [9] Investigating the effectiveness of ... [10] Resources, stressors and psychological ... [11] Effect of forgiveness ... [12] The relationship between ... [13] The effect of cognitive ... [14] Stress management intervention ... [15] The relationship among ... [16] A study of relationship ... [17] Comparison of elderlys "Quality of life" ... [18] Social support as a mediator ... [19] Perceived social support and morale ... [20] Appreciation: Individual differences ... [21] The effectiveness of spiritual ... [22] Hardiness and health ... [23] Stressful life events, personality ... [24] The effect of happiness ... [25] Hope uniquely predicts objective ... [26] The relationship between ... [27] Personality and social resources ... [28] Relationship between perceived ... [29] Type A and ... [30] Relationship between self-efficacy... [31] Dynamic role of social ... [32] A global measure of ... [33] Predicting academic burnout and engagement ... [34] Perceived stress among medical ... [35] The relationship between ... [36] The aggression ... [37] Preliminary study of psychometric ... [38] A Comparison of aggression ... [39] The multidimensional ... [40] Role of religious orientation ... [41] Psychometric properties ... [42] A comparative study of ... [43] The relationship between ... [44] The relationship of hardiness ... [45] The relationship between perceived ... [46] Hardiness and sense of coherence ... [47] The story of hardiness: Twenty... [48] The relationship between ... [49] Prediction strategies to coping ... [50] The relationship between anxiety, depression and perceived social support and quality of life among ... [51] Explaining the relationship ... [52] Comments on trends in hardiness ... [53] The relationship of hardiness ... [54] The Comparative study of ... [55] Relationship between humor ... [56] Correlation between personality traits of parents and type of behavioral disorders in ... 
تجربه مىكنند[4]. از آنجايى كه در دوره بازنشستگى افراد قدرت و و

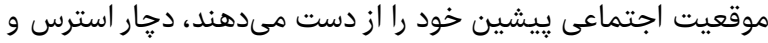

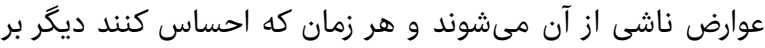

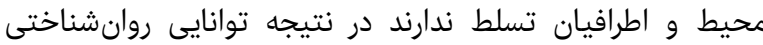

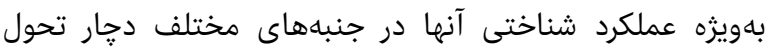

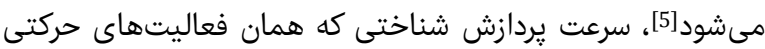

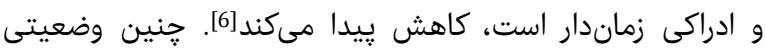

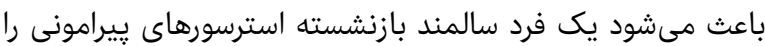

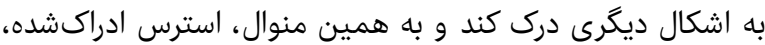

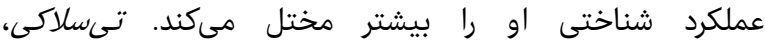

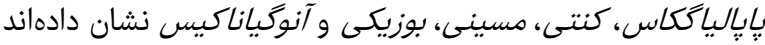

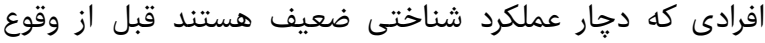

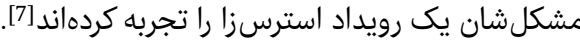

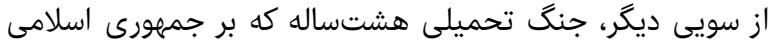

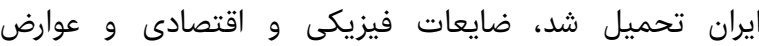

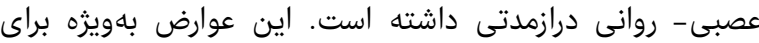

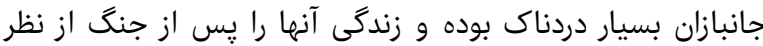

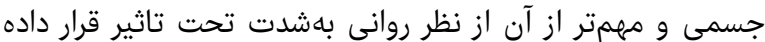

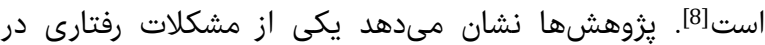

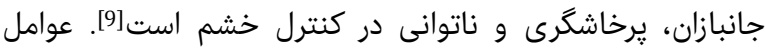

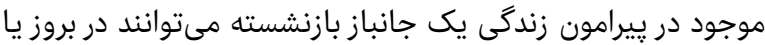

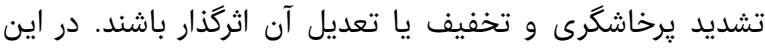

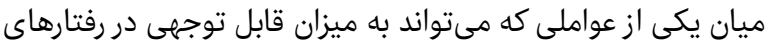

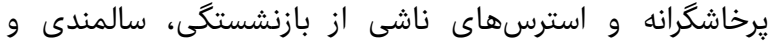

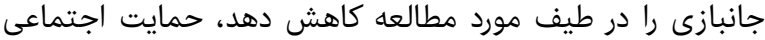
ادراكشده و سخترويى روان شناختى است كان.

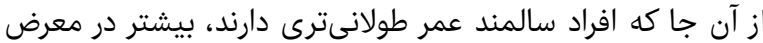

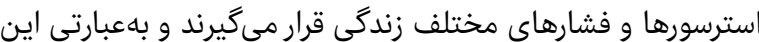

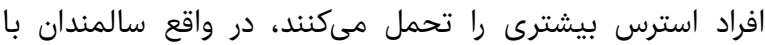

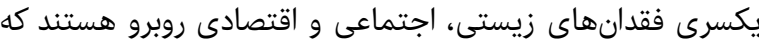

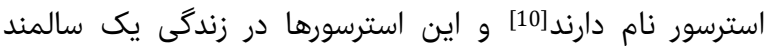

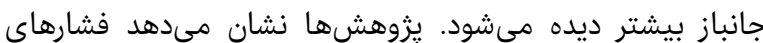

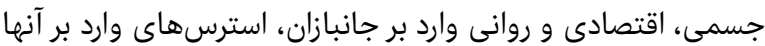

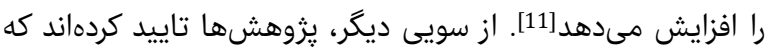

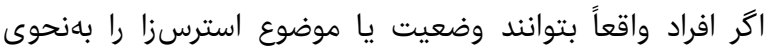

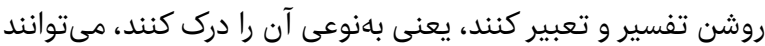

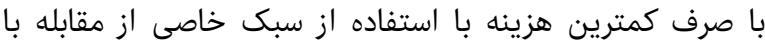

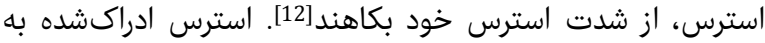

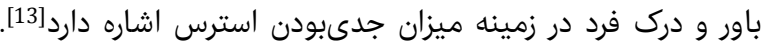

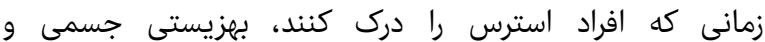

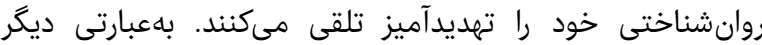

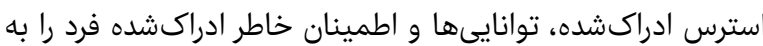

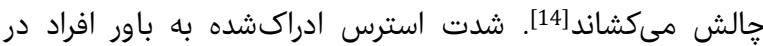

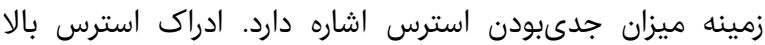

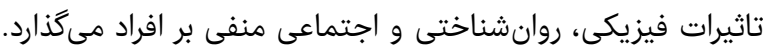

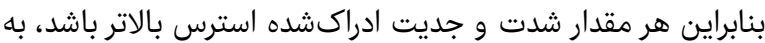

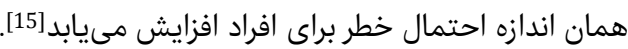

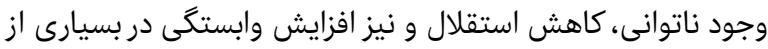

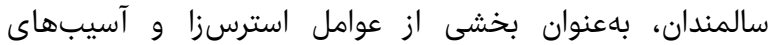

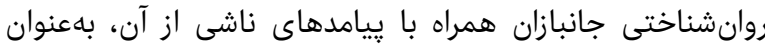

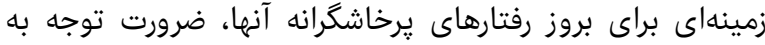

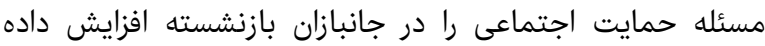

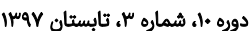

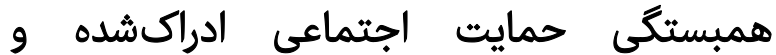

سخترويى روانشناختى با استرس ادراكشده و يرخاشگرى جانبازان بازنشسته روانهي

PhD " لقمان ابراهيمى

كروه روان شناسى، دانشكده علوم انسانى، دانشكاه زنجان، زنجان، ايران

MA فريبا مرادى روانشئ داسن

كروه روانشناسى بالينى، دانشكده روانشناسى، واحد زنجان، دانشكاه آزاد

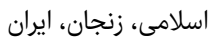

جكيده

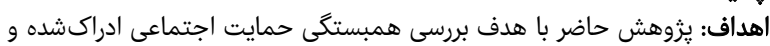

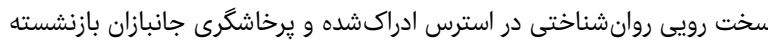

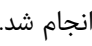

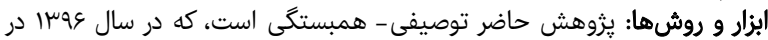

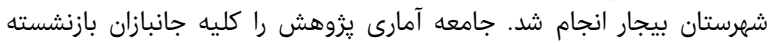

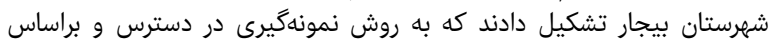

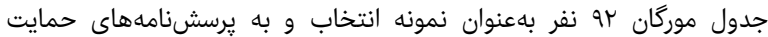

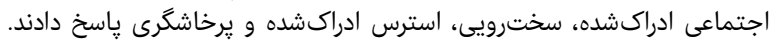

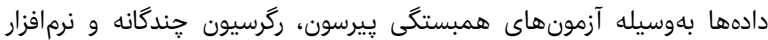
يافئما: SPS 22

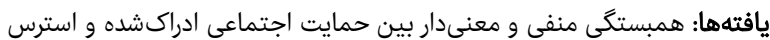

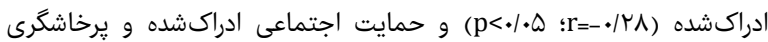

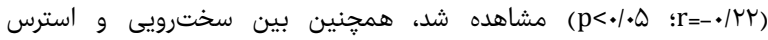

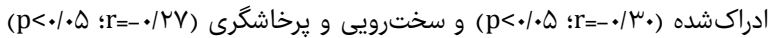

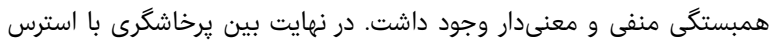

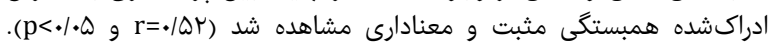

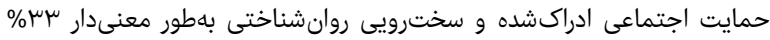

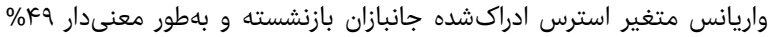

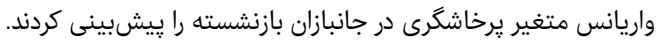

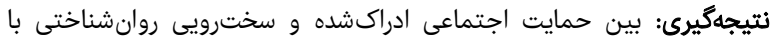

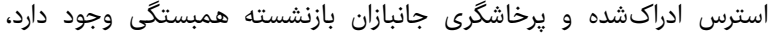

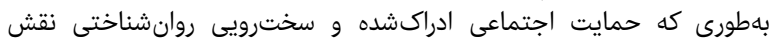

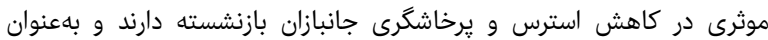

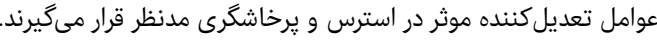

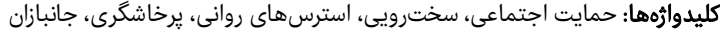

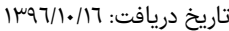

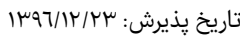

أنويسنده مسئول: l.ebrahimi@znu.ac.ir

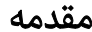

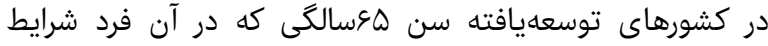

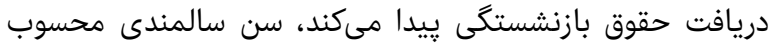

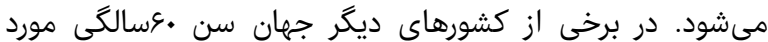

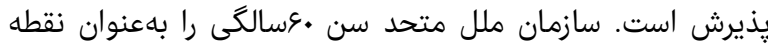

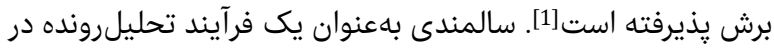

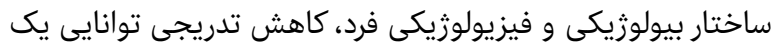

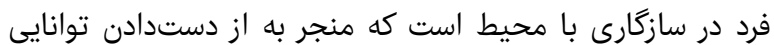

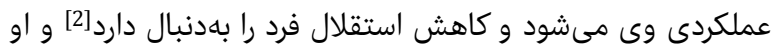

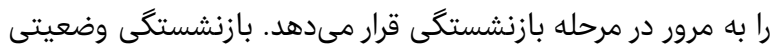

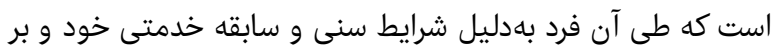

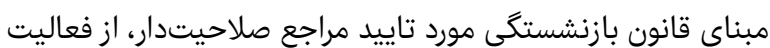

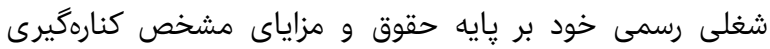

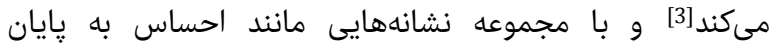

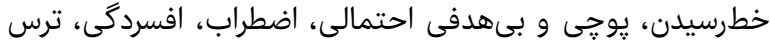
از طردشدن و نكرانىهايى همراه است كه افراد به اشكال مختلفي افتردي، تربن 


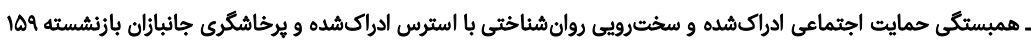

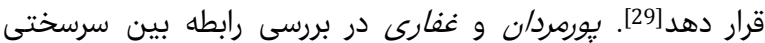

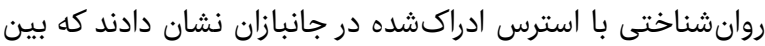

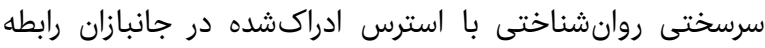

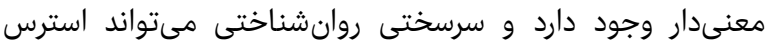

ادراكشده را در جانبازان يِيش وبينى كند [30.

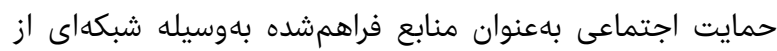

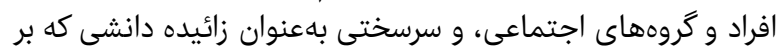

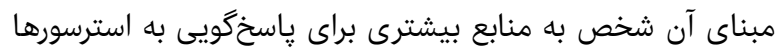

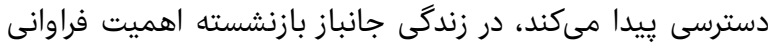

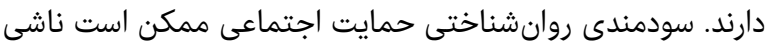

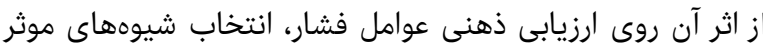

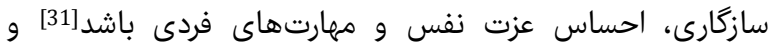

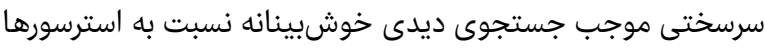

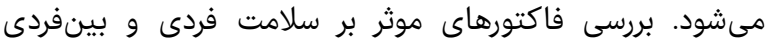

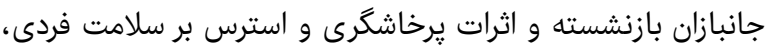

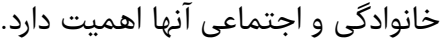

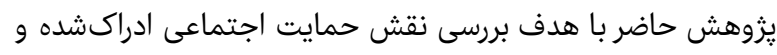

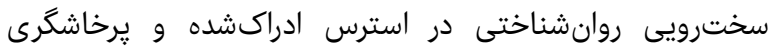

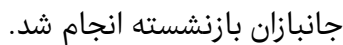

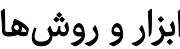

يزوهش حاضر يك يزروهش توصيفى - همبستكى است كه در سال

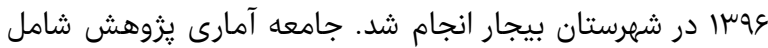

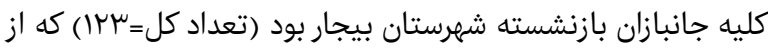

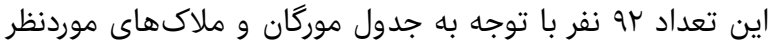

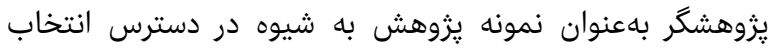

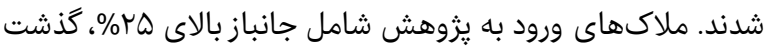

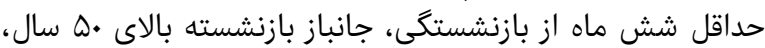

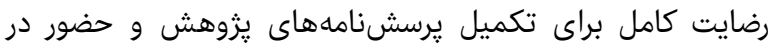

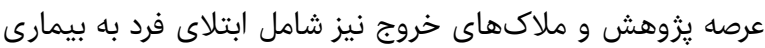

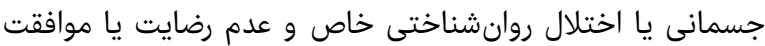

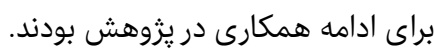

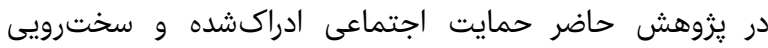

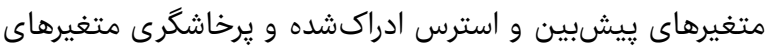

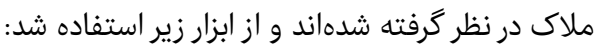

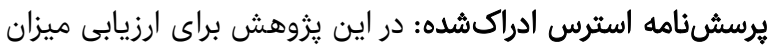

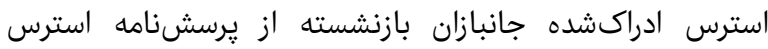

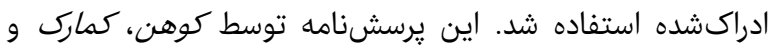

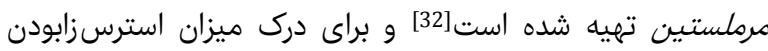

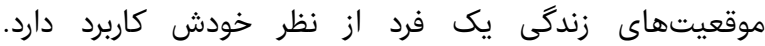

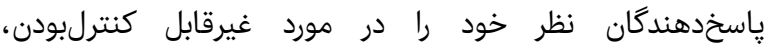

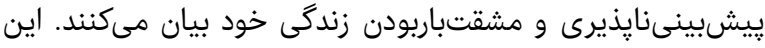

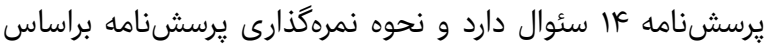

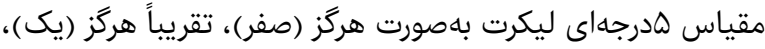

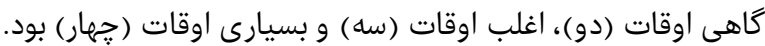

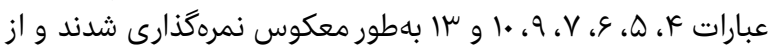

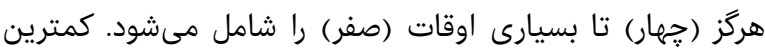

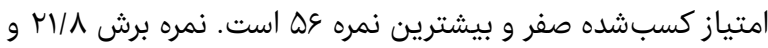

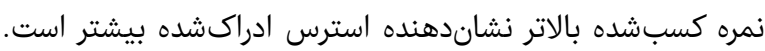

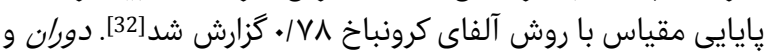

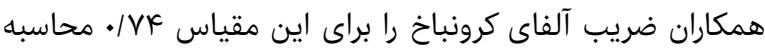

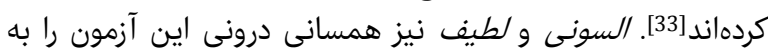

است. حمايت اجتماعى مكانيسمى از ارتباطات ميانفردى است كه إنه

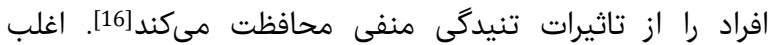

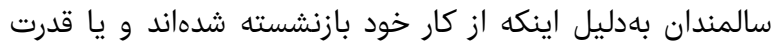

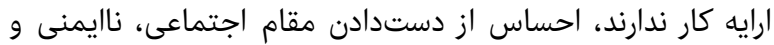

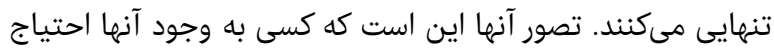

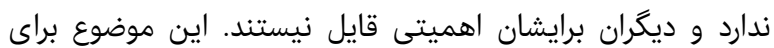

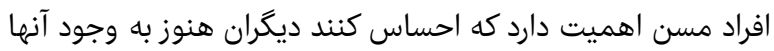

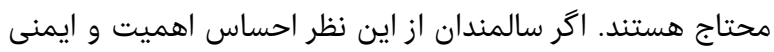

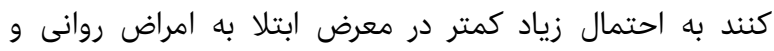

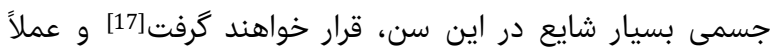

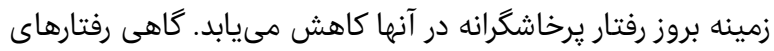

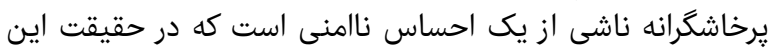

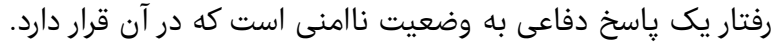

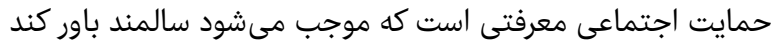

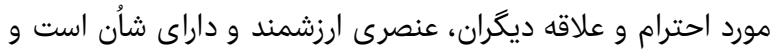

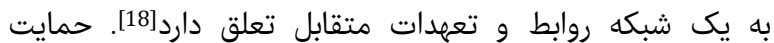

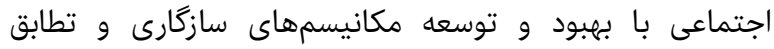

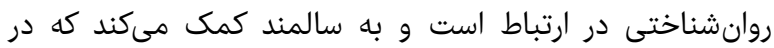

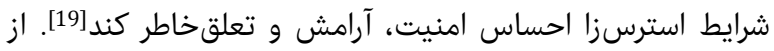

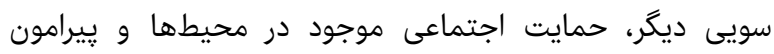

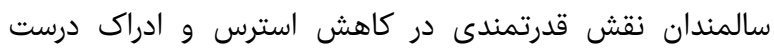

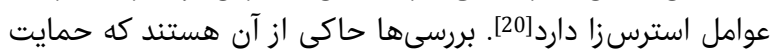

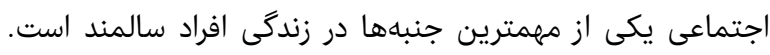

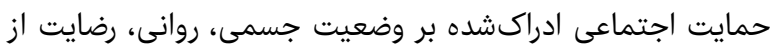

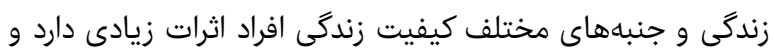

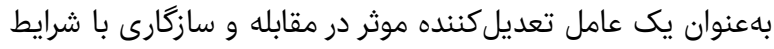

استرسزاى زندگى شناخته شده است [21].

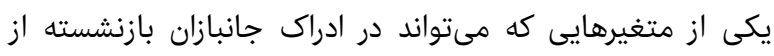

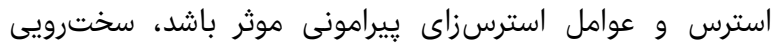

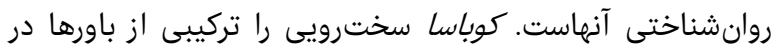

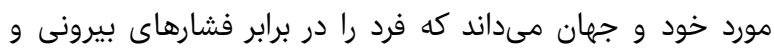

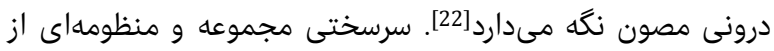

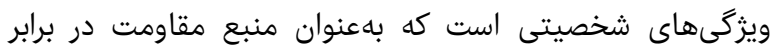

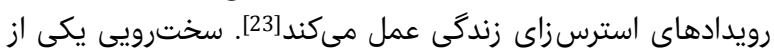

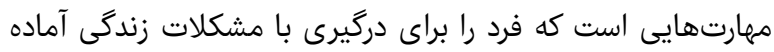

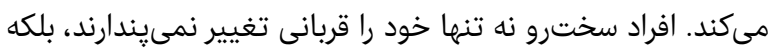

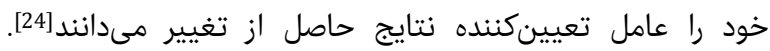

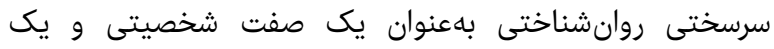

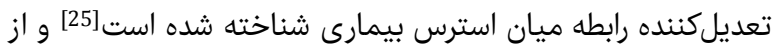

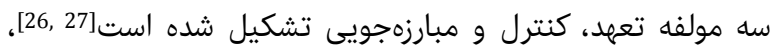

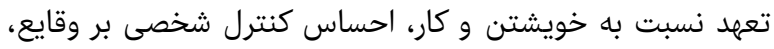

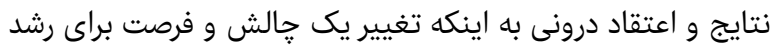

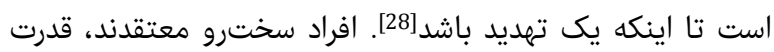

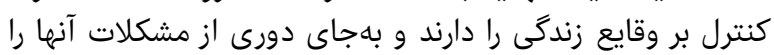

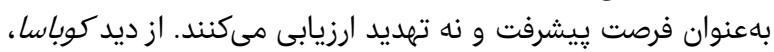

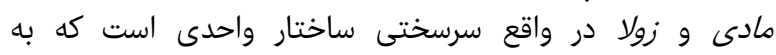

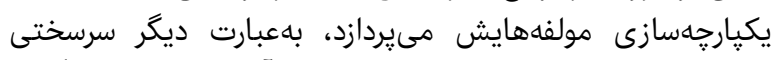

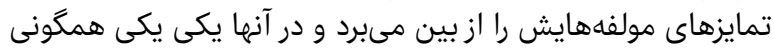

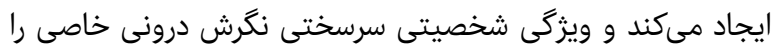

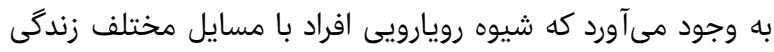

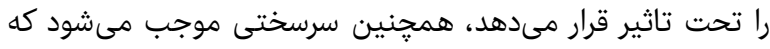
فرد استرسورها را بهصورتى واقع بينانه و با بلندنظرئ مئرى مورد توجه مئ 


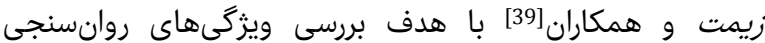

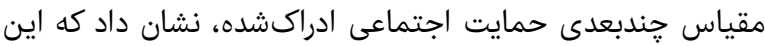

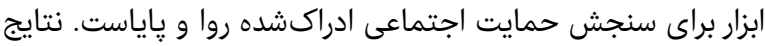

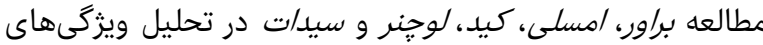

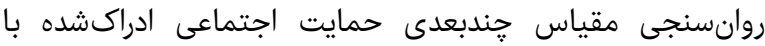

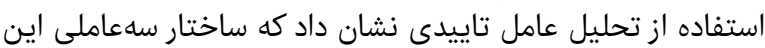

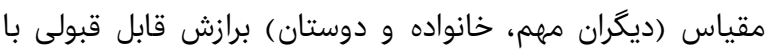

دادهها دارد[41] مقياس (درئ]

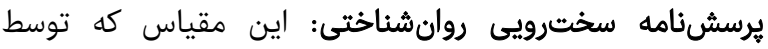

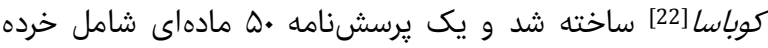

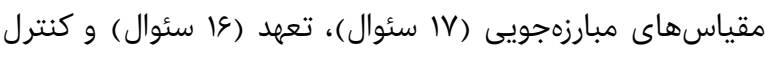

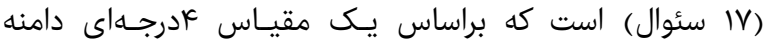

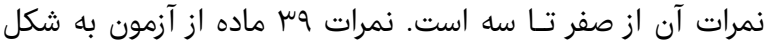

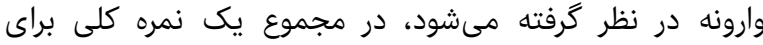

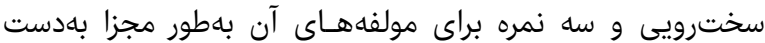

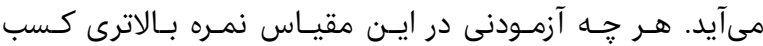

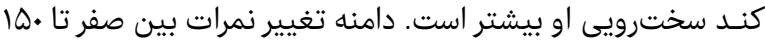

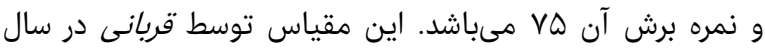

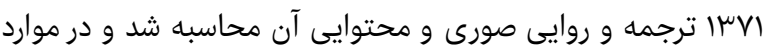

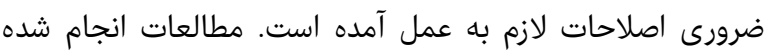

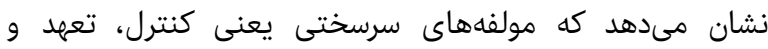

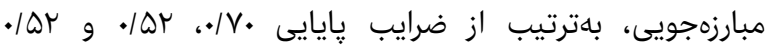

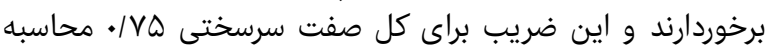

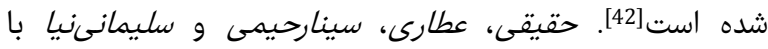

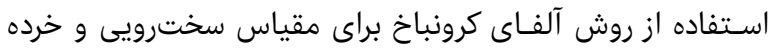

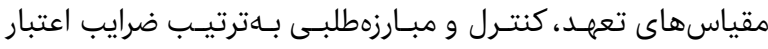

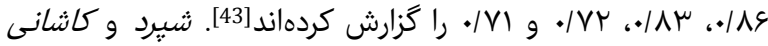

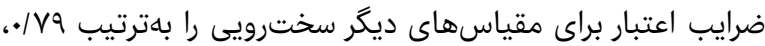

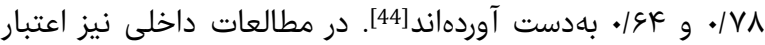

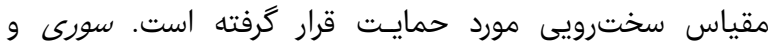

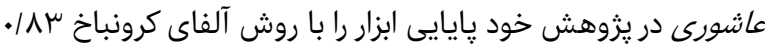

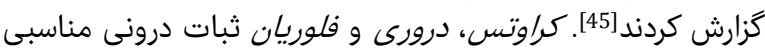

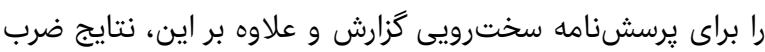

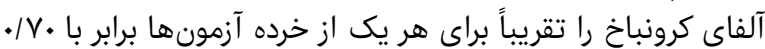

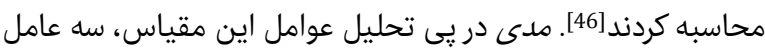

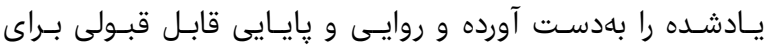

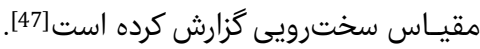

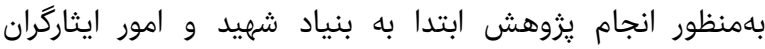

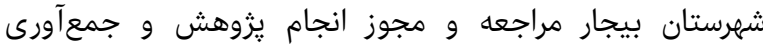

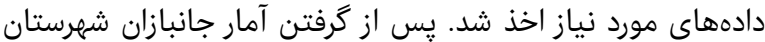

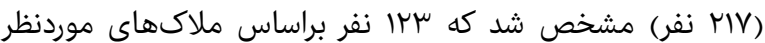

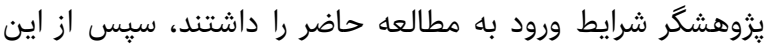

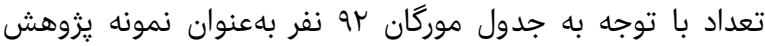

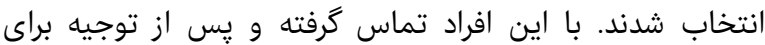

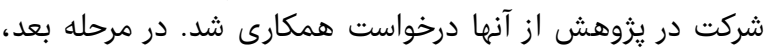

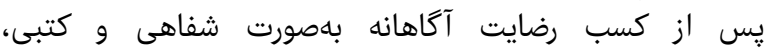

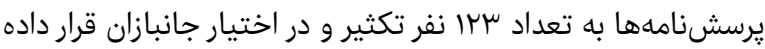

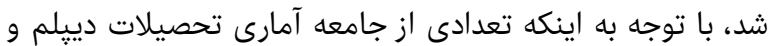

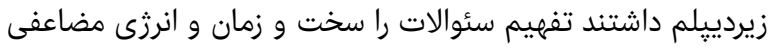

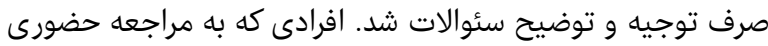

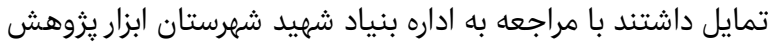

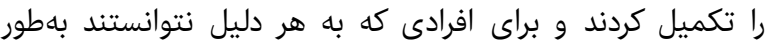

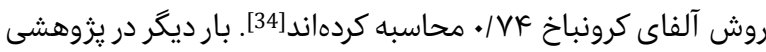

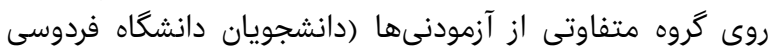

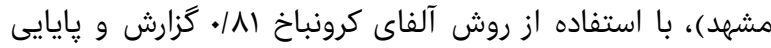

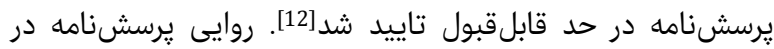

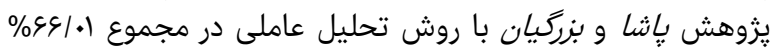

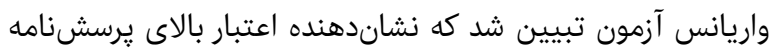

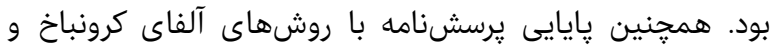

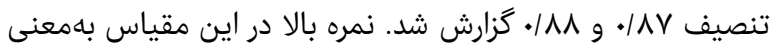

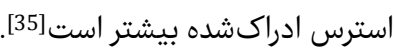

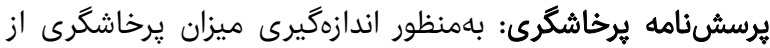

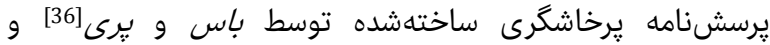

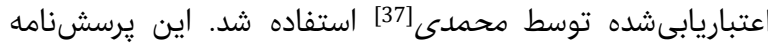

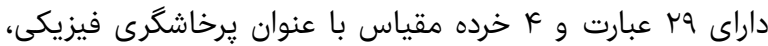

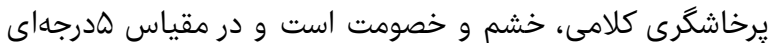

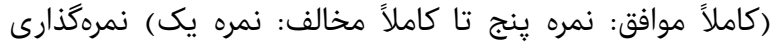

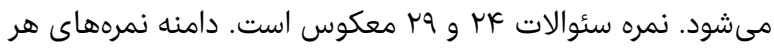

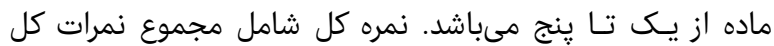

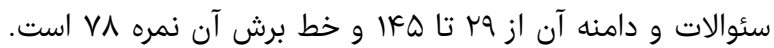

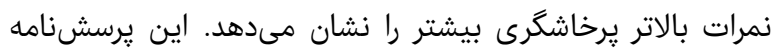

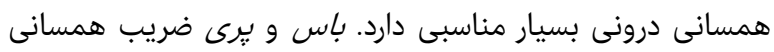

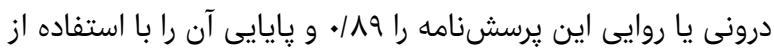

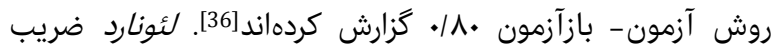

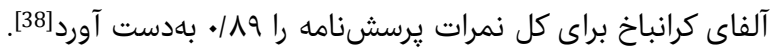

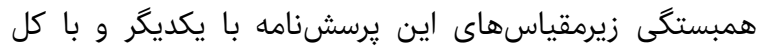

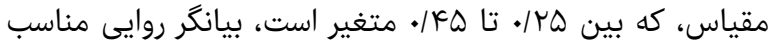

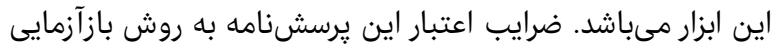

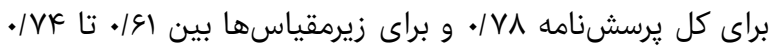

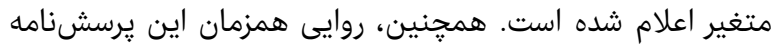

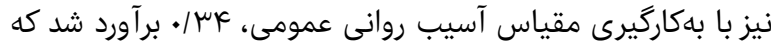

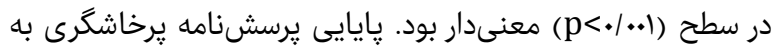

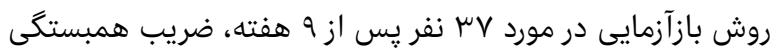

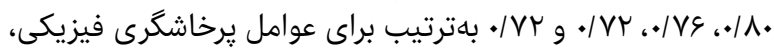

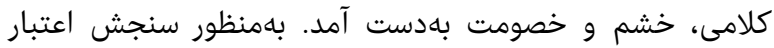

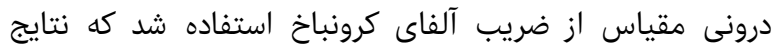

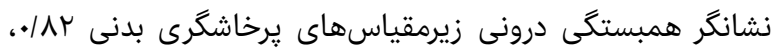

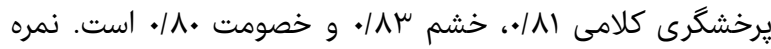

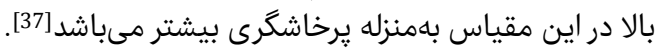

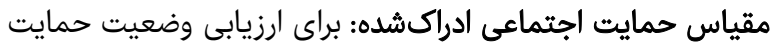

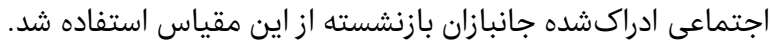

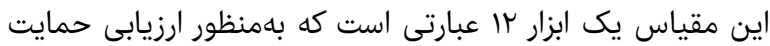

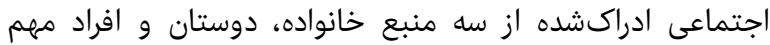

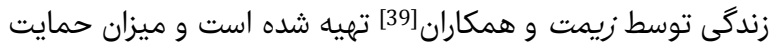

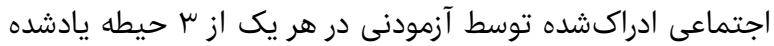

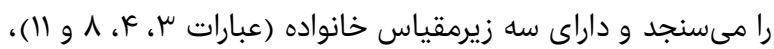

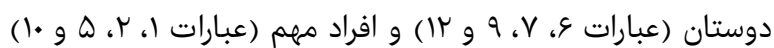

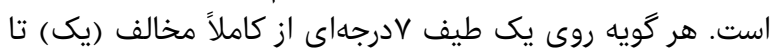

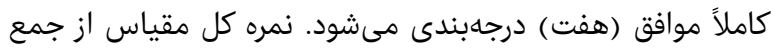

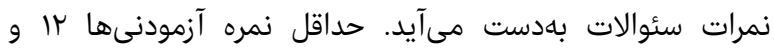

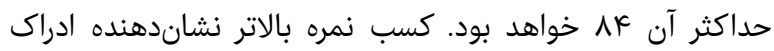

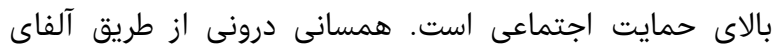

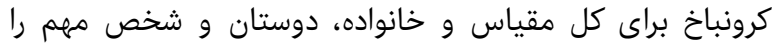

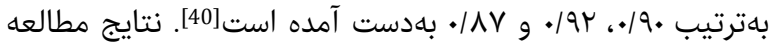




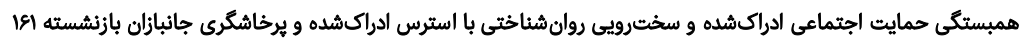

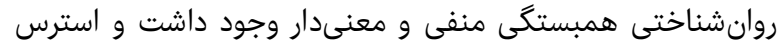

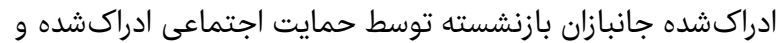

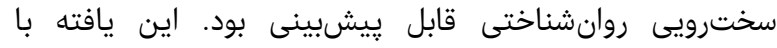

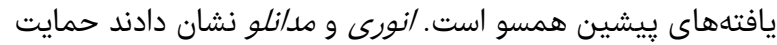

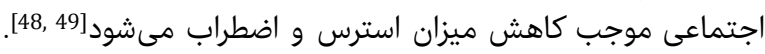

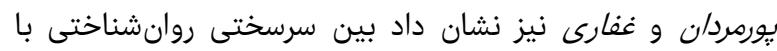

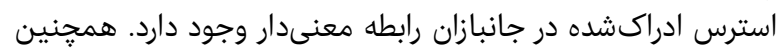

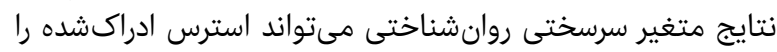

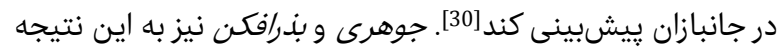

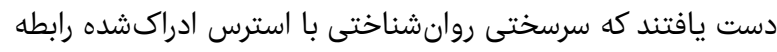

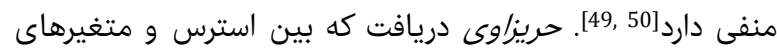

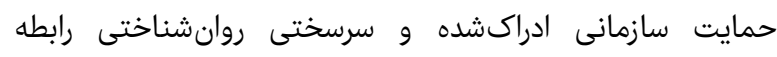

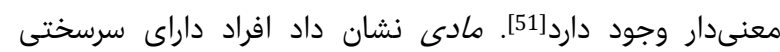

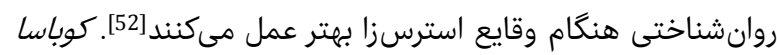

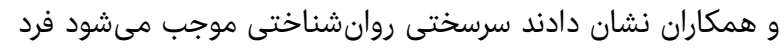

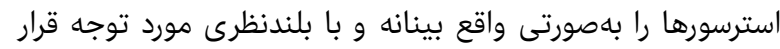

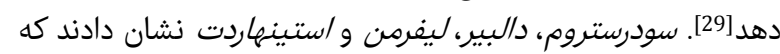

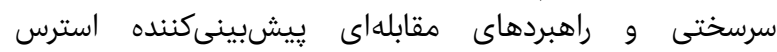

ادراكشده هستند [53].

در تبيين اين يافته مىتوان كفت به به اعتقاد /ريكسون سالمندى كه

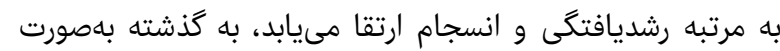

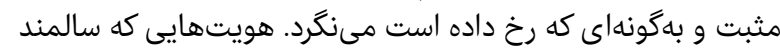

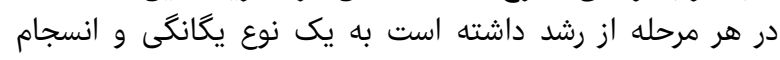

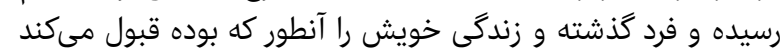

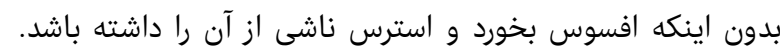

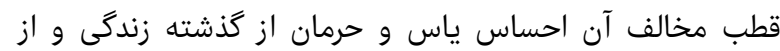

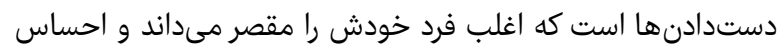

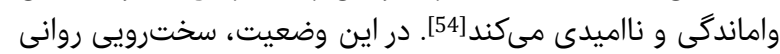

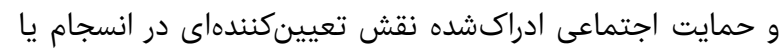

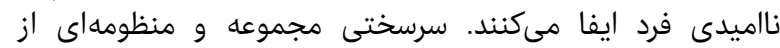

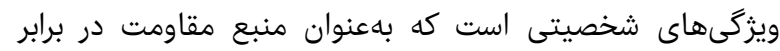

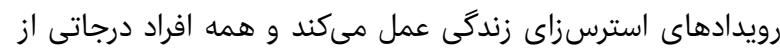

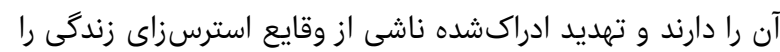

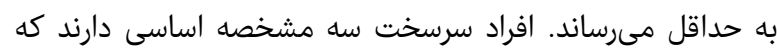

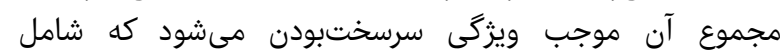

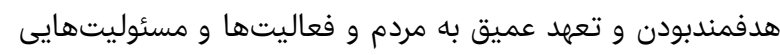

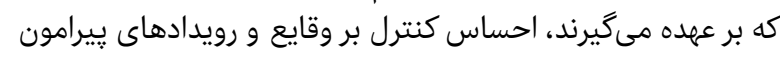

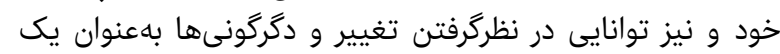

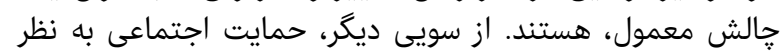

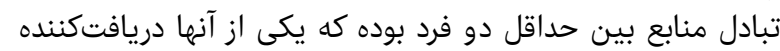

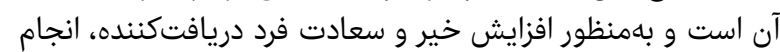

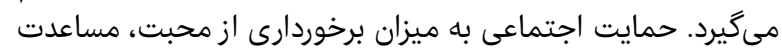

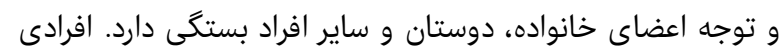

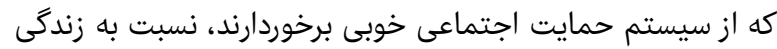

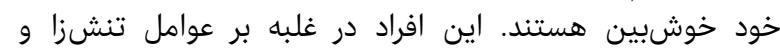

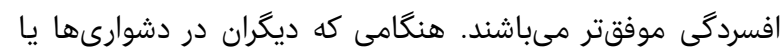

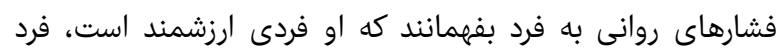

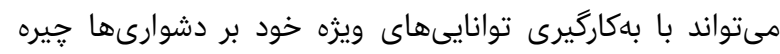

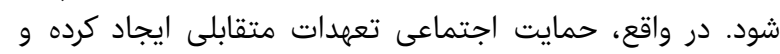

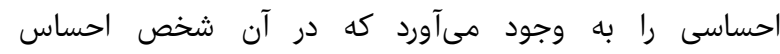

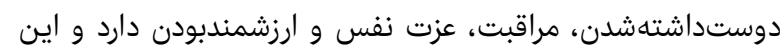

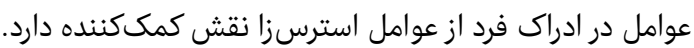

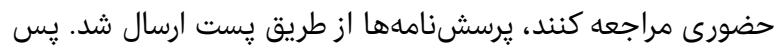

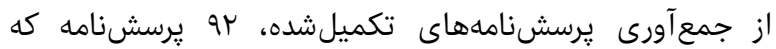

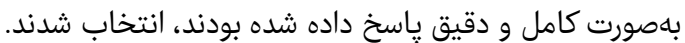

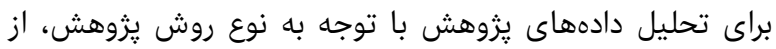

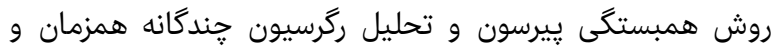

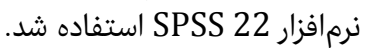

\section{يافتهها}

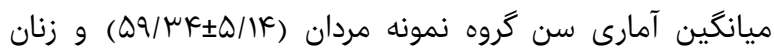

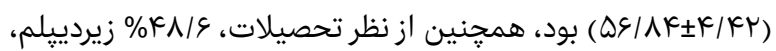

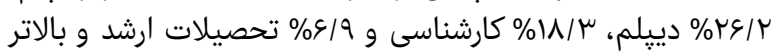

داشتند.

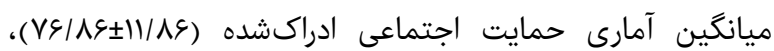

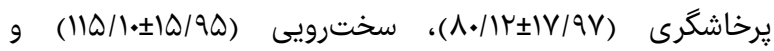

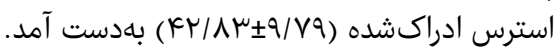

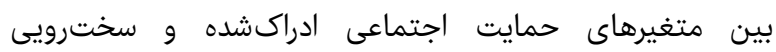

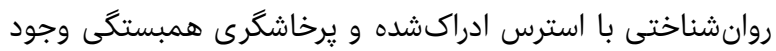

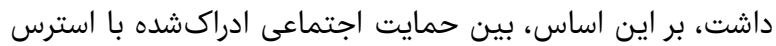

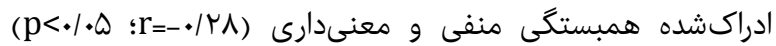

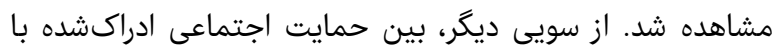

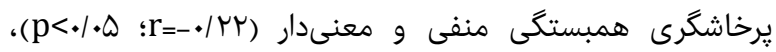

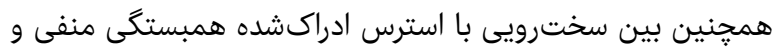

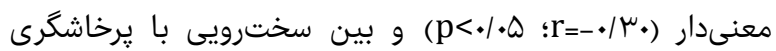

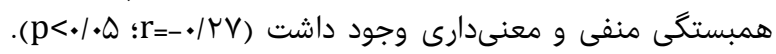

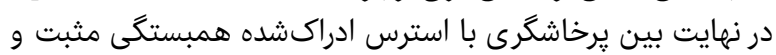

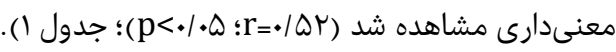

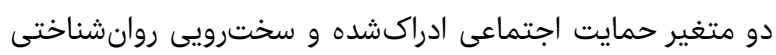

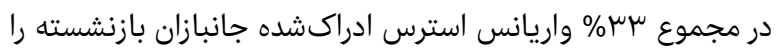

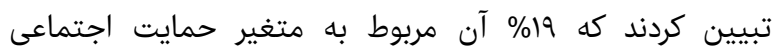

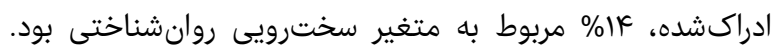

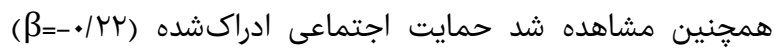

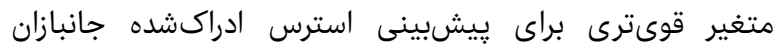

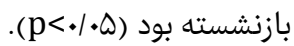

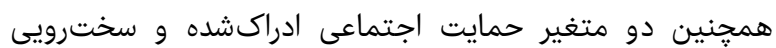

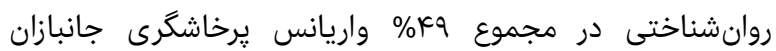

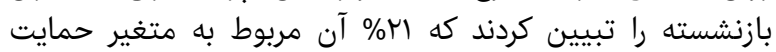

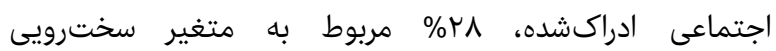

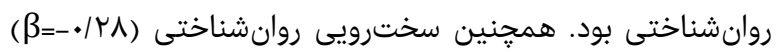

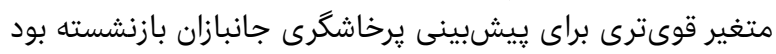

.$(\mathrm{p}<\cdot / \cdot \Delta)$

جدول () ماتريس ضريب همبستخى ييرسون ميان متغيرهاى يزوهش

\begin{tabular}{|c|c|c|c|c|}
\hline \multirow[t]{4}{*}{$k$} & $\mu$ & r & 1 & متغير \\
\hline & & & 1 & 1- حمايت اجتماعى ادراكشده \\
\hline & & 1 & . $/ \mathrm{A}$ & r r- سخترويي \\
\hline & 1 &.$- / \mu$. &.$- / r \Lambda$ & ץ- استرس ادراكشده \\
\hline 1 & •/QT &.$- / T V$ & -r & F- يرخاشكرى \\
\hline
\end{tabular}

يزوهش حاضر با هدف بررسى نقش حمايت اجتماعى ادراكشده و

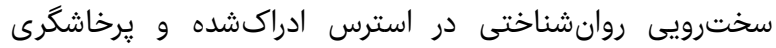

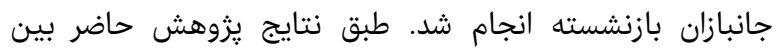

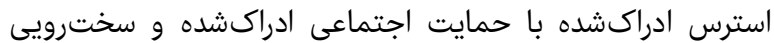




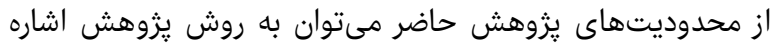

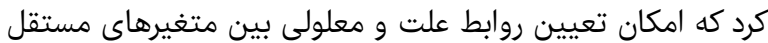

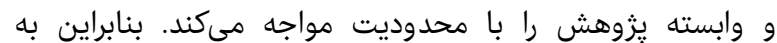

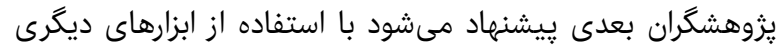

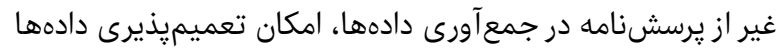

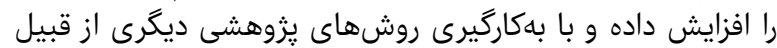

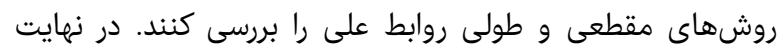

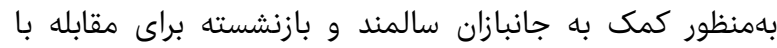

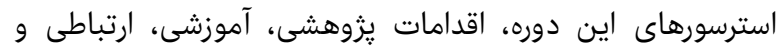

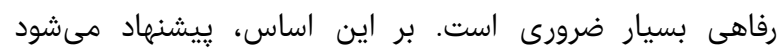

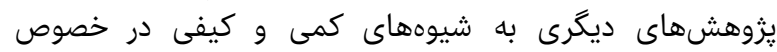

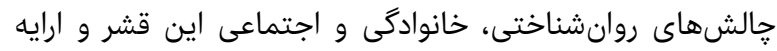
راهكارهاى موثر صورت يذيرد.

\section{نتيجه}

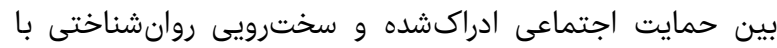

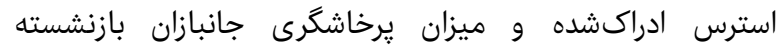

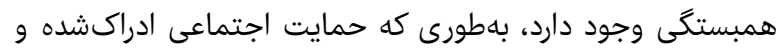

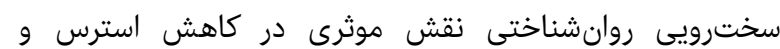

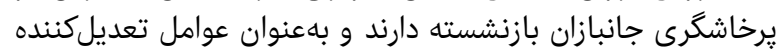
موثر در استرس و يرخاشكرى مدنظر هستند.

تشكر و قدردانى: اين يزوهش در قالب يزوهشى روى برى جانبازان

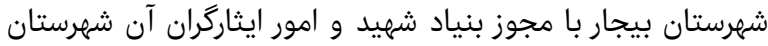

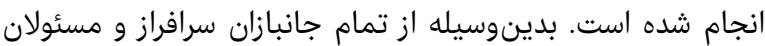

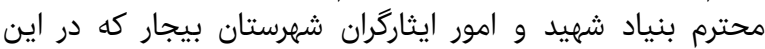
يزوهش مشاركت و همكارى داشتند، سياسكزارى مى بكنيم.

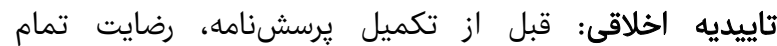

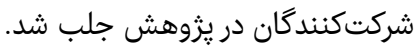

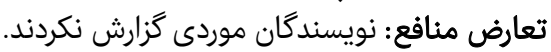

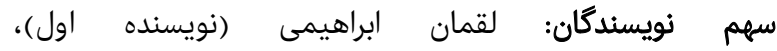

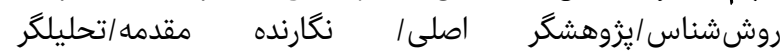

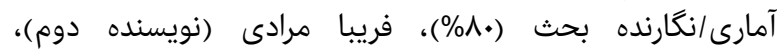

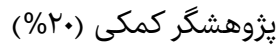

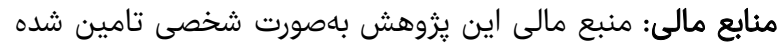

\section{منابع}

1- Momeni Kh, Karimi H. Compare elderly mental health nursing home residents and the non-resident city of Kermanshah. J Kermanshah Univ Med Sci. 2010;14(4):328-35. [Persian]

2- Blackburn JA, Dulmus CN. Handbook of Gerontology: Evidence-based approaches to theory, practice and policy. New Jersey: John Wiley \& Sons; 2007. p. 3.

3- Sajjadi H, Biglryian A. Quality of life in elderly women in Kahrizak Hospice Charity. Payesh. 2006;6(2):105-8. [Persian]

4- Varies MK. The retirement syndrome: The psychology of letting go. Eur Manag J. 2003;21(6):707-16.

5- Yazdkhasti F. A study on cognitive and depressive disorders in the elderly and their correlation with each other. Adv Cogn Sci. 2010;11(4):12-7. [Persian]

6- Christensen H. What cognitive changes can be expected with normal ageing?. Aust N Z J Psychiatry. 2001;35(6):768-75.

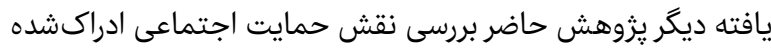

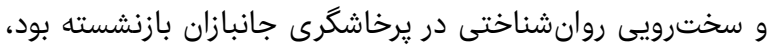

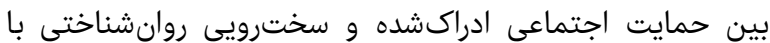

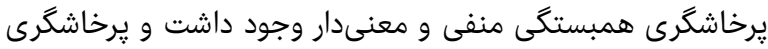

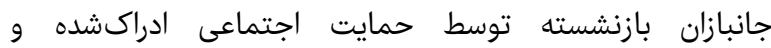

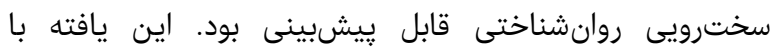

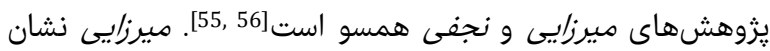

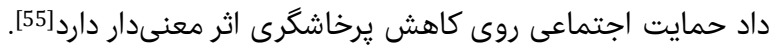

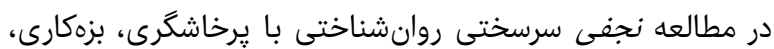

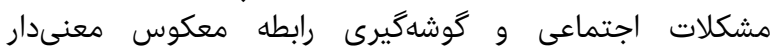

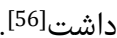

در تبيين يافته يزوهش بايد گفت منظور از دريافت حمايت ميزان

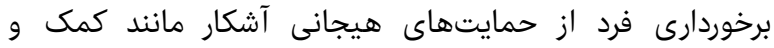

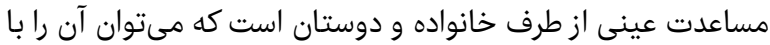

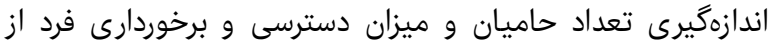

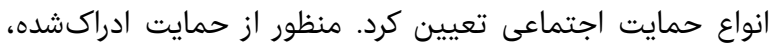

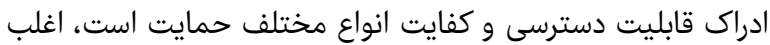

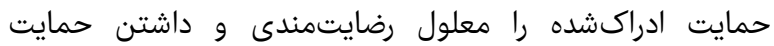

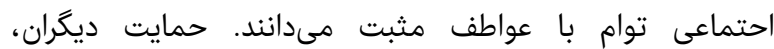

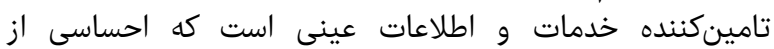

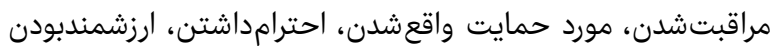

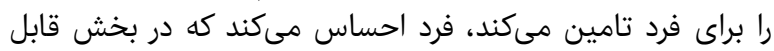

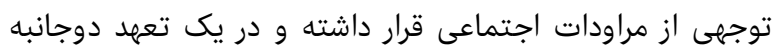

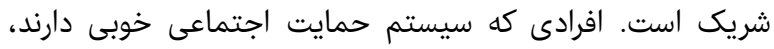

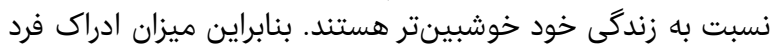

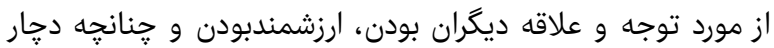

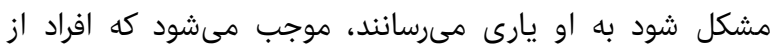

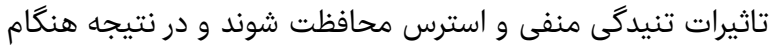

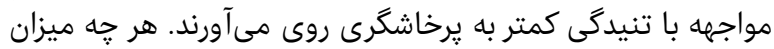

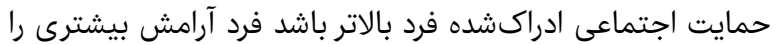

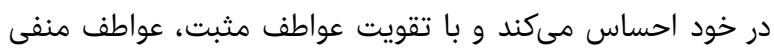

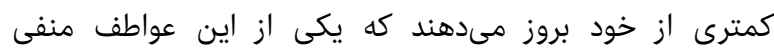

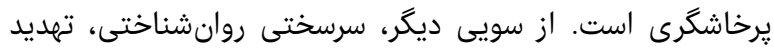

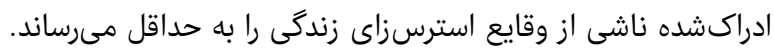

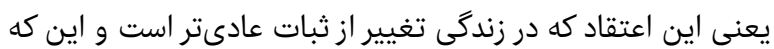

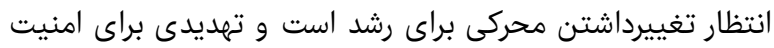

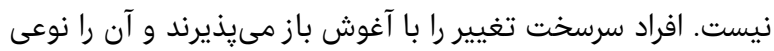

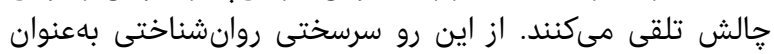

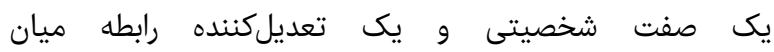

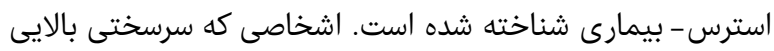

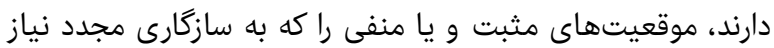

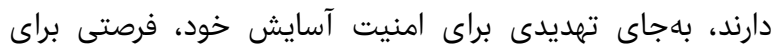

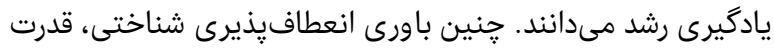

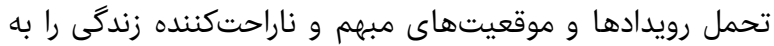

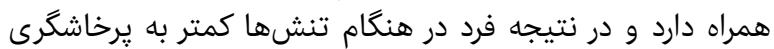

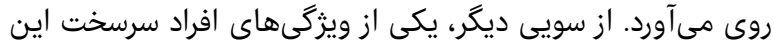

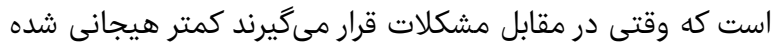

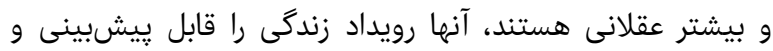

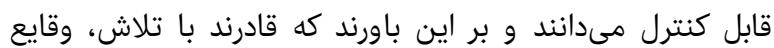

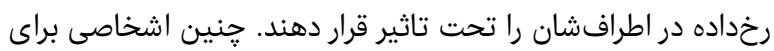

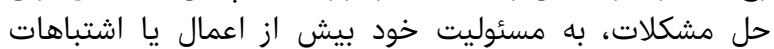

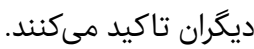




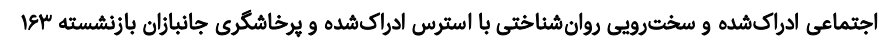
company personnel in Isfahan. J Res Behave Sci. 2015;13(1):134-42. [Persian]

25- Day L, Hanson K, Maltby J, Proctor C, Wood A. Hope uniquely predicts objective academic achievement above intelligence, personality, and previous academic achievement. J Res Personal. 2010;44(4):550-3.

26- Zahed Babolan A, Ghasempour A, Hassanzadeh Sh, Akbari E. The relationship between psychological hardiness and forgiveness and hope in athletes. J Rafsanjan Univ Med Sci. 2012;11(5):449-60. [Persian]

27- Kobasa SC, Puccetti MC. Personality and social resources in stress resistance. J Pers Soc Psychol. 1983;45(4):839-50.

28- Alipour A, Sahraeian MA, Aliakbari M, Haji Aghababai M. Relationship between perceived and hardened social support with mental health and disability status in women with multiple sclerosis. Soc Psychol Res. 2011;1(3):110-33. [Persian]

29- Kobasa SC, Maddi SR, Zola MA. Type A and hardiness. J Behav Med. 1983;6(1):41-51.

30- Sarbanani A, Alimohammadzadeh Kh, Hojjati H. Relationship between self-efficacy and perceived stress in spouses of veterans with post-traumatic stress disorder. Mil Caring Sci. 2017;4(2):102-10. [Persian]

31- Lepore SJ, Evans GW, Schneider ML. Dynamic role of social support in the link between chronic stress and psychological distress. J Pers Soc Psychol. 1991;61(6):899-909.

32- Cohen S, Kamarck T, Mermelstein R. A global measure of perceived stress. J Health Soc Behav. 1983;24(4):38596.

33- Durán A, Extremera N, Rey L, Fernández-Berrocal P, Montalbán FM. Predicting academic burnout and engagement in educational setting: Assessing the Incremental validity of perceived emotional intelligence beyond perceived stress and general self-efficacy. Psicothema. 2006;(18 Suppl):158-64.

34- Al-Sunni A, Latif R. Perceived stress among medical students in preclinical years: A Saudi Arabian perspective. Saudi J Health Sci. 2014;3(3):155-9.

35- Pasha G, Bozorgian R. The relationship between metacognition, perfectionism and self-efficacy, with perceived stress in the students of Azad Ahvaz University. 2011;6(18):91-112. [Persian]

36- Buss AH, Perry M. The aggression questionnaire. J Pers Soc Psychol. 1992;63(3):452-9.

37- Mohammadi N. Preliminary study of psychometric indices of bass and peria's aggression questionnaire. J Soc Sci Humani Shiraz Univ. 2007;25(4):135-51. [Persian]

38- Jenaabadi H, Nastiezaie N. A Comparison of aggression, anxiety, and depression in high school students in Saravan city. J Urmia Nurs Midwifery Fac. 2011;9(3):148-56. [Persian]

39- Zimet GD, Dahlem NW, Zimet SG, Farley GK. The multidimensional scale of perceived social support. J Pers Assess. 1988;52(1):30-41.

40- Sadri Damirchi E, Mohammadi N. Role of religious orientation and perceived social support in prediction of spiritual well-being of veterans. Iran J War Public Health. 2017;9(3):127-31. [Persian]

41- Bruwer B, Emsley R, Kidd M, Lochner C, Seedat S. Psychometric properties of the multidimensional scale of perceived social support in youth. Compr Psychiatry. 2008;49(2):195-201.

42- Bayrami M, EsmailPour Kh, Rezaei Z. A comparative study of hardness and mental health in different levels of
7- Tsolaki M, Papaliagkas V, Kounti F, Messini C, Boziki M, Anogiankis G, et al. Severly stressful events and dementia: A study of an elderly Greek demented population. Psychiatry Res. 2010;176(1):51-4.

8- Mojahed A, Kalantari M, Molavi H, Neshat Doost HT, Bakhshani NM, Shakiba M. Comparative investigation of mental health status of spouses of war handicaps in accordance with husband's disability. Zahedan J Res Med Sci. 2010;12(4):38-42. [Persian]

9- Aghayossefi A, Amirpour B, Alipour A, Zare H. Investigating the effectiveness of cognitive processing in decreasing the anger and hostility of veterans with post traumatic stress disorder. Ebnesina. 2014;16(1-2):6-14. 10- Chokkanathan S. Resources, stressors and psychological distress among older adults in Chennai, India. Soc Sci Med. 2009;68(2):243-50.

11- Ghasemi M, Gholami A, Mehdizadeghan I. Effect of forgiveness training intervention on resilience and perceived stress of veterans' wives. Iran J War Public Health. 2017;9(4):177-183.

12- Besharat MA, Pourang P, Sadeghpour Tabaee A, Pournaghash Tehrani S. The relationship between coping styles and psychological adaptation in the recovery process: Patients with coronary heart disease. Tehran Univ Med J. 2008;66(8):573-9. [Persian]

13- Adavi A, Fathi Marghmalaki R, Madmoli Y. The effect of cognitive-behavioral stress management training on perceived stress in females with hypertension. J Nurs Educ. 2017;6(1):56-62. [Persian]

14- Phillips KM, Antoni MH, Lechner SC, Blomberg BB, Llabre MM, Avisar E, et al. Stress management intervention reduces serum cortisol and increases relaxation during treatment for Nonmetastatic breast cancer. Psychosom Med. 2008;70:1044-9.

15- Spada M, Georgiou GA, Wells A. The relationship among metacognition, attention control and state anxiety. Cogn Behav Ther. 2009;39(1):64-71.

16- Aghayari hir T, Ghasemzadeh D, Ebrahimi Orang A. A study of relationship between social support and healthrelated quality of life among elderly people in Tabriz. Iran Nurs Sci Assoc. 2017;2(1):20-7. [Persian]

17- Hesamzadeh A, Seyyed Madah SB, Mohammadi Shahabolaghi F, Fallahi Khoshkanb M, Rahgozar M. Comparison of elderlys "Quality of life" living at homes and in private or public nursing homes. Iran J Ageing. 2010;4(4):66-74. [Persian]

18- Cobb S. Social support as a mediator of life stress. Psychosom Med. 1976;38:300-14.

19- Yoo YG. Perceived social support and morale of the elderly staying at home. Taehan Kanho Hakhoe Chi. 2004;34(2):297-306. [Korean]

20- Adler MG, Fagley NS. Appreciation: Individual differences in finding value and meaning as a unique predictor of subjective well-being. J Pers. 2005;73(1):79114.

21- Sedaghat Ghotbabadi S, Haji Alizadeh K. The effectiveness of spiritual-religion psychotherapy on mental distress (depression, anxiety and stress) in the elderly living in nursing homes. Health Spiritual Med Ethics. 2018;5(1):20-5.

22- Kobasa SC, Maddi SR, Kahn. Hardiness and health: A prospective study. J Pers Soc Psychol. 1982;42(1):168-77. 23- Kobasa SC. Stressful life events, personality, and health: An inquiry into hardiness. J Pers Soc Psychol. 1979;37(1):1-11.

24- Abedi MR, Afshar H, Jafari E, Nikneshan Sh. The effect of happiness training on general health of oil refining 
50- Sadoughi M, Hesampour F. The relationship between anxiety, depression and perceived social support and quality of life among hemodialysis patients. J Clin Nurs Midwifery. 2017;6(3):56-71. [Persian]

51- Nasiri Valik Bani F, Abdolmaleki Sh. Explaining the relationship between perceived social support and quality of life, perceived stress mediator role in female-headed households in Sanandaj. J Appl Soc. 2016;27(4):99-116. [Persian]

52- Maddi SR. Comments on trends in hardiness research and theorizing. Consult Psychol J Pract Res. 1999;51(2):67-71.

53- Soderstrom M, Dolbier C, Leiferman J, Steinhardt M. The relationship of hardiness, coping strategies, and perceived stress to symptoms of illness. J Behav Med. 2000;23(3):311-28.

54- Jouzy P, Ranjbari M, Moradi A, Zarei F .The Comparative study of positive emotion, creativity and psychological well-being in evolution stage of middle age and adolescence. J Aging Psychol. 2016;1(3):187-99. [Persian]

55- Khodabakhshi-Koolaee A, MirAfzal NS. Relationship between humor and social support with sex satisfaction in elderly married women. Iran Nurs Sci Assoc. 2017;2(1):1-10. [Persian]

56- Najmi SB, Hassanzadeh A. Correlation between personality traits of parents and type of behavioral disorders in children. J Fundam Ment Health. 2010;12(2):522-33. [Persian] religiosity in culturalists in Khoy. I Educ Sci. 2012;5(19):75-90. [Persian]

43- Rahimian Boogar E, Asgharnejad Farid AA. The relationship between psychological hardiness also egoresiliency and mental health in adolescent and adult survivors of Bam earthquake. Iran J Psychiatry Clin Psychol. 2008;14(1):62-70. [Persian]

44- Sheppard JA, Kashani JH. The relationship of hardiness, gender, and stress to health outcomes in adolescents. J Pers. 1991;59(4):747-68.

45- Souri A, Ashoori J. The relationship between perceived social support, psychological hardiness and family communication patterns with quality of life among patients with type II diabetes. J Diabetes Nurs. 2015;3(2):53-65.

46- Kravetz S, Drory Y, Florian V. Hardiness and sense of coherence and their relation to negative affect. Eur J Personal.1993;7(4):233-44.

47- Maddi SR. The story of hardiness: Twenty years of theorizing, research, and practice. Consult Psychol J Pract Res. 2002;54(3):173-85.

48- Gheibizadeh M, Pourghane P, Mosaffa Khomami H, Heidari F, Atrkar Roushan Z. The relationship between stressors and coping strategies employed by retired elderly. Iran J Psychiatr Nurs. 2016;4(5):36-43. [Persian] 49- Bayrami M, Zahmatyar H, Khosroshahi J. Prediction strategies to coping with stress in the pregnancy women with first experience on the based factors hardiness and social support. Iran J Nurs Res. 2012;7(27):1-9. [Persian] 